\title{
Systems Pharmacological Approach to the Effect of Bulsu-san Promoting Parturition
}

\author{
Su Yeon Suh and Won G. An \\ Department of Pharmacology, School of Korean Medicine, Pusan National University, Yangsan, Gyeongnam 50612, Republic of Korea \\ Correspondence should be addressed to Won G. An; wgan@pusan.ac.kr
}

Received 28 July 2017; Accepted 25 September 2017; Published 29 October 2017

Academic Editor: Gihyun Lee

Copyright (C) $2017 \mathrm{Su}$ Yeon Suh and Won G. An. This is an open access article distributed under the Creative Commons Attribution License, which permits unrestricted use, distribution, and reproduction in any medium, provided the original work is properly cited.

\begin{abstract}
Bulsu-san (BSS) has been commonly used in oriental medicine for pregnant women in East Asia. The purpose of this research was to elucidate the effect of BSS on ease of parturition using a systems-level in silico analytic approach. Research results show that BSS is highly connected to the parturition related pathways, biological processes, and organs. There were numerous interactions between most compounds of BSS and multiple target genes, and this was confirmed using herb-compound-target network, target-pathway network, and gene ontology analysis. Furthermore, the mRNA expression of relevant target genes of BSS was elevated significantly in related organ tissues, such as those of the uterus, placenta, fetus, hypothalamus, and pituitary gland. This study used a network analytical approach to demonstrate that Bulsu-san (BSS) is closely related to the parturition related pathways, biological processes, and organs. It is meaningful that this systems-level network analysis result strengthens the basis of clinical applications of BSS on ease of parturition.
\end{abstract}

\section{Introduction}

The name of Bulsu-san (BSS) originated from its therapeutic effects that help to promote easy labor as if being touched by merciful Buddha's hand [1]. BSS is composed of Angelicae Sinensis Radix (Danggui, DG) and Cnidium officinale Makino (Cheongung, CG), which is one of the most commonly used herb pairs in Traditional Medicine of East Asia and the usual component ratio is $2: 3$ (CG:DG) or $1: 1$ [2]. BSS is widely used in women's medicine in East Asia; its recognized therapeutic effects are as follows: removal of impure blood, blood making, easy parturition, acceleration of labor, elimination of dead fetus or placenta, amelioration of pain, nourishing blood, and promoting blood circulation [3].

What is more, recent experimental research on the CGDG herb pair indicated that they affect the nourishment of blood [4], activate blood circulation, and prevent blood stasis [5]. In addition, the CG-DG herb pair showed significant inhibitory effects on the proliferation and protein synthesis of vascular smooth muscle cells [6]. It was suggested BSS could affect the activities of Akt kinase and eNOS by increasing intracellular $\mathrm{Ca}^{2+}$ and reducing ROS levels [7] and regulate menstruation and provide relief from pain by enabling the management of uterine smooth muscle contractions [8]. Although BSS has therapeutic effects on various pathological symptoms in pregnant or childbearing aged women, this research focused on the molecular mechanisms and impact of BSS on easing parturition and the acceleration of labor.

In terms of parturition onset, numerous studies have described the complex hormone interactions between estrogen, progesterone, oxytocin, corticosteroid, and prostaglandin. Among these, corticotrophin releasing hormone (CRH) is regarded as a trigger that initiates the labor [9]. The placenta releases substantial amounts of $\mathrm{CRH}$, which stimulates the pituitary glands of both mother and fetus to secrete adrenocorticotropin hormone [10]. This in turn induces the release of estrogen precursor, which is converted into estrogen by the placenta that induces smooth muscle contraction [10]. Additionally, dilatation of cervical connective tissue and smooth muscle is induced by the following changes: a shift from progesterone to estrogen dominance, increased responsiveness to oxytocin via the upregulation of myometrial oxytocin receptor, increased prostaglandins synthesis in uterus, increased myometrial gap junction formation, decreased nitric oxide activity, and increased influx of calcium into myocyte [11]. 


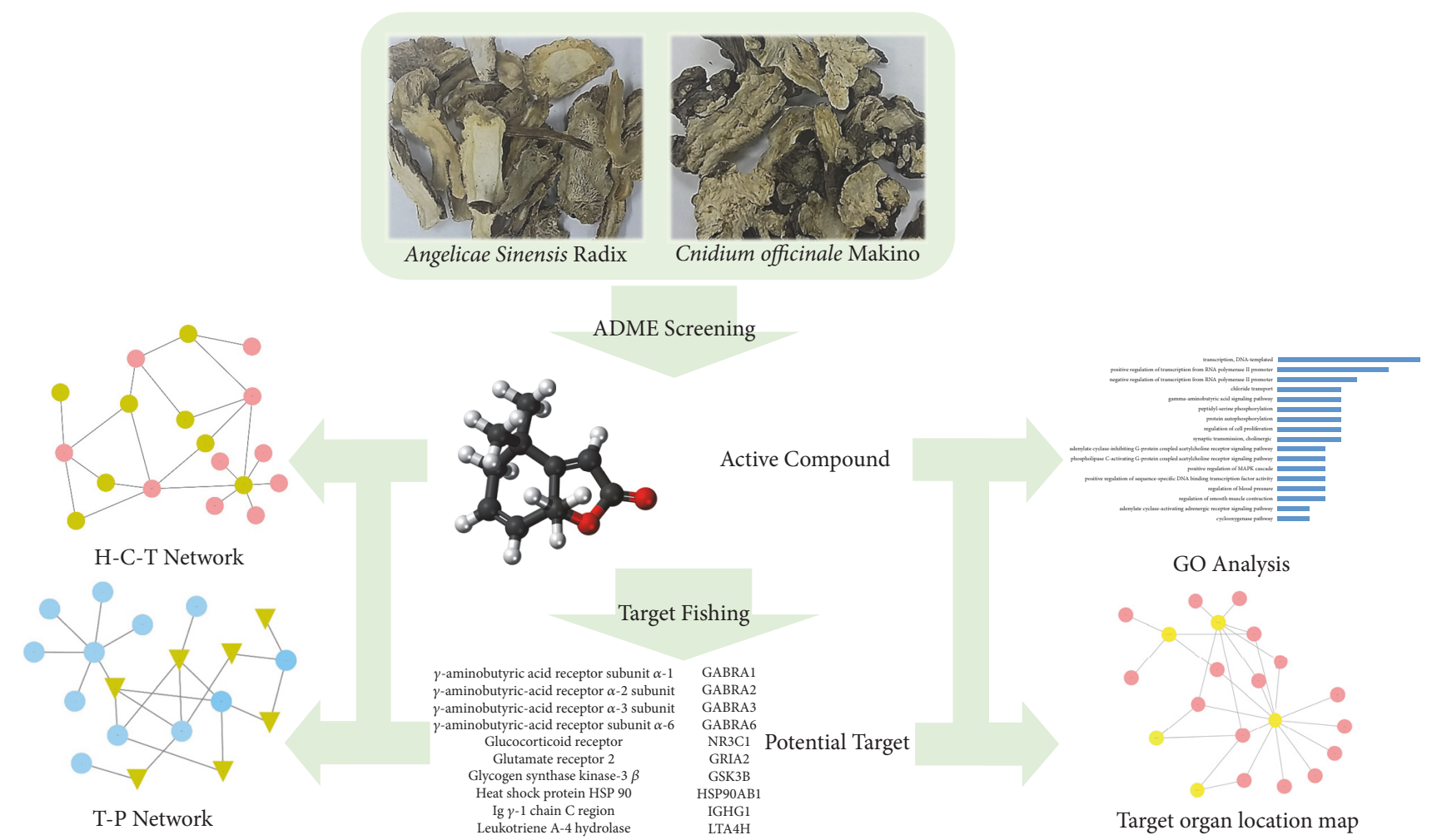

FIGURE 1: The workflow: the network pharmacological approach of Bulsu-san (BSS), namely, active compounds screening, target fishing, network analysis, and relevant organ location mapping was performed in this study.

The hypothesis of this study was that BSS may promote the positive-feedback of hormone loops as well as a series of myometrial and cervical changes to ease parturition and safely accelerate labor. A network based in silico approach was used to identify the effect of BSS on parturition related systems and the aim of this study was to elucidate the effect of BSS on the parturition by system-level analysis. The workflow of the network pharmacological study is summarized in Figure 1.

\section{Material and Methods}

2.1. Identification of Active Compounds. Compounds in CG and DG were identified using a phytochemical database that is the Traditional Chinese Medicine Systems Pharmacology (TCMSP, http://ibts.hkbu.edu.hk/LSP/tcmsp.php). We applied parameters related to absorption, distribution, metabolism, and excretion (ADME), namely, human druglikeness (DL) [12], oral bioavailability (OB) [13], and Caco2 permeability (Caco-2) to screen the Potential active compounds in BSS [14].

2.1.1. Drug-Likeness Evaluation. DL helps filter "drug-like" compounds in oriental herbs, as DL represents a qualitative concept for valuations based on how "drug-like" a prospective compound is [15]. Accordingly, a high DL may lead to a greater possibility of therapeutic success, and compounds with a higher DL value are more likely to possess certain biological properties [16]. The calculations of DL in TCMSP database were based on Tanimoto coefficient formula [17] as follows:

$$
F(A, B)=\frac{A \times B}{A^{2}+B^{2}-A \times B},
$$

where $A$ represents the molecular parameters of herbal compounds and $B$ is the average molecular parameters of all compounds in the Drugbank database (http://www.drugbank.ca/) [18]. In the present study, we excluded compounds with a DL of $<0.08$. Other previous researches of herbal formulas set a higher threshold in the range of 0.1 to 0.18 . However, we found out that most compounds of DG have low DL. In detail, only 36 compounds of 125 in DG show higher or equal DL value than 0.08 . For this reason, this study sets a lower threshold of DL than other previous researches to see the most potential targets of BSS.

2.1.2. Oral Bioavailability (OB) Prediction. OB is defined as the ratio of active compounds' absorption into the systemic circulation, which represents the convergence of the ADME process [13]. OB values are dependent on drug dissolution in the gastrointestinal (GI) tract and hepatic and intestinal firstpass metabolism, as well as on intestinal membrane permeation, which makes it a major pharmacokinetic parameter for drug evaluations [16]. In this study, the OB threshold was set as $\geq 15 \%$.

2.1.3. Caco-2 Permeability Screening. Caco-2 permeability is used to predict the absorption of an orally administered 
drug [14]. Surface absorptivity of the small intestine is maximized with the presence of villi and microvilli, for this reason most orally administered drug absorption occurs in the small intestine [19]. Moreover, the movement of orally administered drugs across the intestinal epithelial barrier determines the rate and extent of human absorption and ultimately affects drug bioavailability [20]. In the present study, compounds with OB, DL and Caco-2 values of greater than $15 \%, 0.08$, and $>-0.4$, respectively, were regarded as active compounds and subjected to further analysis.

2.1.4. Lipinskis Rule (LR) Screening. In addition, the screening standard used was defined based on Lipinski's rule (LR), which identifies druggable compounds as having molecular weight (MW) of $\leq 500 \mathrm{Da}(\mathrm{MW} \leq 500)$, chemical composition with $\leq 5$ hydrogen-bond donors, $\leq 10$ hydrogen-bond acceptors, and an octanol-water partition coefficient, AlogP of $\leq$ 5 [21]. AlogP can be used to estimate local hydrophobicity, to produce molecular hydrophobicity maps, and to evaluate hydrophobic interactions in protein-ligand complexes [22]. Hdon and Hacc are the number of possible hydrogen-bond donors and acceptors, and the hydrogen-bonding capacity of a drug solute is recognized as a crucial determinant of permeability; moreover high hydrogen-bonding potential is often related to low permeability and absorption [23]. Eventually, in the present study, we selected active compounds satisfying the following criteria: $\mathrm{OB} \geq 15 \%$; $\mathrm{DL} \geq 0.08$; Caco- $2 \geq-0.4$; MW $\leq 500$; H-bond donors $\leq 5$; H-bond acceptors $\leq 10$; $A \log \mathrm{P} \leq 5$.

2.2. Target Fishing. Aside from filtering active compounds, we also sought to identify the molecular targets of these active compounds. Compound-target interaction profiles were established based on a systematic prediction of multiple drug-target interactions tool which employs random forest (RF) and support vector machine (SVM) methods and integrates chemical, genomic, and pharmacological information for drug targeting and discovery on a large scale [24]. Compound-target interactions satisfying SVM score $\geq 0.8$ and RF score $\geq 0.7$ were selected for further study. Additionally, filtered compound-target interaction profile mapping was performed using the UniProt database (http://www.uniprot.org/) [25].

2.3. Gene Ontology (GO) Analysis. Biological process (BP) of gene ontology (GO) analysis was employed to determine the biological properties of target genes [26]. GO annotation indicates the possibility of direct statistical analysis on gene function information. In this research, GO BP terms with $P$ values $<0.01$ were employed and the data was collected using the DAVID 6.8 Gene Functional Classification Tool (http://david.abcc.ncifcrf.gov/).

2.4. Network Construction and Analysis. In order to understand the multiscale interactions between the active compounds of BSS and targets, two types of networks were built: (1) the herb-compound-target network (H-C-T network), in which nodes represent either compounds, target genes, or herbs and edges indicate herb-compoundtarget connections; and (2) the target-pathway network (T-P network) to extract the pathways from KEGG database (http://www.genome.jp/kegg/), and the terms highly associated with parturition with $P$ values $<0.05$ were selected as the related pathways of targets in this work. Related targets were mapped onto relevant pathways, which resulted in the T-P network. Both networks were generated in Cytoscape 3.5.1, an open-source biological network visualization and data integration software package [27].

2.5. Target Organ Location Map. Tissue-specific patterns of mRNA expression can indicate important associations with biological events or gene functions [28]. To explore the beneficial effects of BSS during parturition, it is important that the tissue mRNA expression profiles of target genes at the organ level be known [29]. The target organ location map was built according to the Dataset: GeneAtlas U133A, gcrma (http://biogps.org). BioGPS database provides expression data acquired by direct measurements of gene expression obtained by microarrays analysis [30]. First, the mRNA expression patterns of each target gene in 176 parts of organ tissues were obtained. Second, average values were calculated for each gene. Third, frequency of above average mRNA expression tissue organs was inspected. Forth, based on the result from the third step and parturition mechanism theory, mRNA expression data of relevant organ tissues were extracted and categorized into 6 groups, namely, uterus and/or uterus corpus, fetus and/or placenta, hypothalamus and/or pituitary, smooth muscle, and whole blood.

\section{Results}

3.1. Identification of Active Compounds. 314 compounds of BSS were identified, including 189 molecules in CG and 125 in DG (as shown in Supplementary Material Table S1 in Supplementary Material available online at https://doi.org/10.1155/ 2017/7236436) and active compounds met the criteria OB $\geq 15 \%$, Caco- $2 \geq-0.4$, and DL $\geq 0.08$, as well as the standards of Lipinski's rule (LR) (as shown in Table 1). In detail, 60 active compounds were initially chosen, but 8 compounds were present in both herbs, namely, 3-butylidene7-hydroxyphthalide, adenine, $\mathrm{BdPh}$, beta-selinene, palmitic acid, senkyunolide-C, senkyunolide-D, and senkyunolide$\mathrm{E}$, and 14 had no target protein information and were thus excluded from the list of active compounds, whereas 27 compounds with lower ADME properties than above thresholds were included, which were reported to be related to oxytocin. In total, 65 active compounds were filtered.

Although ligustilide and ferulic acid have a DL of $<0.08$, both were included in this study. Since ligustilide (C12, DL = $0.07, \mathrm{OB}=53.72$, Caco-2 $=1.3$ ) was reported to be the main compound of DG in uterine contraction [31], and ferulic acid $(\mathrm{C} 42, \mathrm{DL}=0.06, \mathrm{OB}=54.97, \mathrm{Caco}-2=0.53)$ has been reported to be useful for the treatment of vascular diseases $[6,32]$ and blood deficiency syndrome [33] in China and to suppress inflammatory responses and tumor progression [34]. Some other compounds also have been shown experimentally to have various biological activities; for example, crysophanol $(\mathrm{C} 42, \mathrm{DL}=0.21, \mathrm{OB}=18.64, \mathrm{Caco}-2=0.62)$ can be used to treat menorrhagia and thrombocytopenia [35]. Perlolyrine 
TABLE 1: 65 Potential active compounds of BSS (compound with $*$ was present in both herbs).

\begin{tabular}{|c|c|c|c|c|c|}
\hline ID & Active compounds & OB (\%) & Caco-2 & $\mathrm{DL}$ & Herb \\
\hline $\mathrm{C} 1$ & ()-alpha-Terpineol & 46.3 & 1.28 & 0.03 & DG \\
\hline $\mathrm{C} 2$ & ()-Aromadendrene & 55.74 & 1.81 & 0.1 & CG \\
\hline $\mathrm{C} 3$ & ()-Terpinen-4-ol & 81.41 & 1.36 & 0.03 & CG \\
\hline $\mathrm{C} 4$ & (+)-alpha-Funebrene & 52.87 & 1.79 & 0.1 & CG \\
\hline C5 & $(+)$-Ledol & 16.96 & 1.43 & 0.12 & DG \\
\hline C6 & (1R,5R,7S)-4,7-Dimethyl-7-(4-methylpent-3-enyl)bicyclo[3.1.1] hept-3-ene & 16.23 & 1.86 & 0.09 & CG \\
\hline $\mathrm{C} 7$ & $\begin{array}{c}\text { (1S,4aR,8aR)-1-Isopropyl-7-methyl-4-methylene-2,3,4a,5,6,8a-hexahydro-1H- } \\
\text { naphthalene }\end{array}$ & 19.8 & 1.86 & 0.08 & DG \\
\hline $\mathrm{C} 8$ & (1S,4E,8E,10R)-4,8,11,11-tetramethylbicyclo[8.1.0] undeca-4,8-diene & 21.69 & 1.86 & 0.08 & CG \\
\hline C9 & (3E)-3-butylidene-7-hydroxy-2-benzofuran-1-one & 42.17 & 1.03 & 0.08 & DG \\
\hline $\mathrm{C} 10$ & (L)-alpha-Terpineol & 48.8 & 1.39 & 0.03 & CG \\
\hline C11 & (R)-Linalool & 39.8 & 1.33 & 0.02 & CG \\
\hline $\mathrm{C} 12$ & (Z)-Ligustilide & 53.72 & 1.3 & 0.07 & CG \\
\hline $\mathrm{C} 13$ & 1-Acetyl-beta-carboline & 67.12 & 1.18 & 0.13 & CG \\
\hline $\mathrm{C} 14$ & 1-beta-Ethylacrylate-7-aldehyde-beta-carboline & 28.53 & 0.45 & 0.31 & CG \\
\hline $\mathrm{C} 15$ & $\begin{array}{l}\text { 1H-Cycloprop(e)azulen-7-ol, decahydro-1,1,7-trimethyl-4-methylene-, } \\
\text { (1aR-(1aalpha,4aalpha,7beta,7abeta,7balpha))- }\end{array}$ & 82.33 & 1.37 & 0.12 & CG \\
\hline $\mathrm{C} 16$ & 1-Terpineol & 49.83 & 1.24 & 0.03 & CG \\
\hline $\mathrm{C} 17$ & 2,6-Di(phenyl)thiopyran-4-thione & 69.13 & 1.74 & 0.15 & DG \\
\hline $\mathrm{C} 18$ & 2-[(2S,5S,6S)-6,10-Dimethylspiro[4.5] dec-9-en-2-yl]propan-2-ol & 37.62 & 1.44 & 0.09 & CG \\
\hline $\mathrm{C} 19^{*}$ & 3-Butylidene-7-hydroxyphthalide & 62.68 & 1 & 0.08 & CG\&DG \\
\hline $\mathrm{C} 20$ & 4,7-Dihydroxy-3-butylphthalide & 106.09 & 0.69 & 0.1 & CG \\
\hline $\mathrm{C} 21$ & 49070_FLUKA & 85.51 & 1.29 & 0.12 & CG \\
\hline $\mathrm{C} 22$ & 4-Hydroxy-3-butylphthalide & 70.31 & 0.9 & 0.08 & CG \\
\hline $\mathrm{C} 23$ & 58870_FLUKA & 49.01 & 1.82 & 0.1 & CG \\
\hline $\mathrm{C} 24^{*}$ & Adenine & 62.81 & -0.3 & 0.03 & CG\&DG \\
\hline $\mathrm{C} 25$ & $\mathrm{ADO}$ & 15.98 & -1.56 & 0.18 & CG \\
\hline $\mathrm{C} 26$ & alpha-Cubebene & 16.73 & 1.83 & 0.11 & CG \\
\hline $\mathrm{C} 27$ & alpha-Selinene & 31.81 & 1.82 & 0.1 & CG \\
\hline $\mathrm{C} 28$ & Aromadendrene oxide 2 & 65.1 & 1.56 & 0.14 & CG \\
\hline $\mathrm{C} 29^{*}$ & $\mathrm{BdPh}$ & 42.44 & 1.32 & 0.07 & CG\&DG \\
\hline $\mathrm{C} 30$ & beta-Chamigrene & 31.99 & 1.82 & 0.08 & DG \\
\hline $\mathrm{C} 31^{*}$ & beta-Selinene & 24.39 & 1.83 & 0.08 & CG\&DG \\
\hline $\mathrm{C} 32$ & beta-Cubebene & 32.16 & 1.82 & 0.11 & CG \\
\hline $\mathrm{C} 33$ & Cadinene & 17.12 & 1.88 & 0.08 & DG \\
\hline $\mathrm{C} 34$ & Caffeic acid & 25.76 & 0.21 & 0.05 & CG \\
\hline $\mathrm{C} 35$ & Carotol & 149.03 & 1.46 & 0.09 & CG \\
\hline $\mathrm{C} 36$ & Cedrene & 51.14 & 1.82 & 0.11 & CG \\
\hline $\mathrm{C} 37$ & Chuanxiongol & 22.19 & 0.94 & 0.1 & CG \\
\hline C38 & cis-Thujopsene & 56.43 & 1.84 & 0.12 & DG \\
\hline C39 & Coniferyl ferulate & 4.54 & 0.71 & 0.39 & DG \\
\hline $\mathrm{C} 40$ & Crysophanol & 18.64 & 0.62 & 0.21 & CG \\
\hline C41 & FA & 68.96 & -1.5 & 0.71 & CG \\
\hline $\mathrm{C} 42$ & Ferulic acid (CIS) & 54.97 & 0.53 & 0.06 & DG \\
\hline $\mathrm{C} 43$ & $\begin{array}{c}\text { InChI }=1 / \mathrm{C} 15 \mathrm{H} 24 / \mathrm{cl}-10-7-8-15-9-12(10) 14(3,4) 13(15) 6-5-11(15) 2 / \mathrm{h} 7,11-13 \mathrm{H}, 5-6,8- \\
9 \mathrm{H} 2,1-4 \mathrm{H}\end{array}$ & 55.56 & 1.79 & 0.1 & DG \\
\hline $\mathrm{C} 44$ & L-Bornyl acetate & 65.52 & 1.29 & 0.08 & CG \\
\hline $\mathrm{C} 45$ & Methyl palmitate & 18.09 & 1.37 & 0.12 & CG \\
\hline $\mathrm{C} 46$ & Myricanone & 40.6 & 0.67 & 0.51 & CG \\
\hline $\mathrm{C} 47$ & Nicotinic acid & 47.65 & 0.34 & 0.02 & DG \\
\hline
\end{tabular}


TABLE 1: Continued.

\begin{tabular}{lcccccc}
\hline ID & Active compounds & OB (\%) & Caco-2 & DL & Herb \\
C48 & Oleic acid & 33.13 & 1.17 & 0.14 & CG \\
C49* & Palmitic acid & 19.3 & 1.09 & 0.1 & CG\&DG \\
C50 & Perlolyrine & 65.95 & 0.88 & 0.27 & CG \\
C51 & PLO & 14.07 & 0.69 & 0.43 & CG \\
C52 & Scopoletol & 27.77 & 0.71 & 0.08 & DG \\
C53 & Senkyunolide A & 26.56 & 1.3 & 0.07 & CG \\
C54 & Senkyunolide G & 39.52 & 0.63 & 0.08 & CG \\
C55* & Senkyunolide-C & 46.8 & 0.87 & 0.08 & CG\&DG \\
C56 & Senkyunolide-D & 79.13 & 0.12 & 0.1 & CG\&DG \\
C57* & Senkyunolide-E & 34.4 & 0.55 & 0.08 & CG\&DG \\
C58 & Senkyunolide-K & 61.75 & 0.52 & 0.08 & CG \\
C59 & Sinapic acid & 64.15 & 0.48 & 0.08 & CG \\
C60 & Sphingomyelin & 0.31 & -0.46 & 0.51 & DG \\
C61 & Stearic acid & 17.83 & 1.15 & 0.14 & CG \\
C62 & Stigmasterol & 43.83 & 1.44 & 0.76 & DG \\
C63 & Succinic acid & 29.62 & -0.44 & 0.01 & DG \\
C64 & Sucrose & 7.17 & -2.89 & 0.23 & CG \\
C65 & Wallichilide & 42.31 & 0.82 & 0.71 & CG \\
\hline & & & &
\end{tabular}

$(\mathrm{C} 52, \mathrm{DL}=0.27, \mathrm{OB}=65.95, \mathrm{Caco}-2=0.88)$ was confirmed to have a protective effect on injured human umbilical vein endothelial cells [36], and myricanone (C48, DL $=0.51, \mathrm{OB}$ $=57.61$, Caco- $2=0.67)$ was found to best inhibit mouse skin tumor progression [37].

3.2. Target Fishing. The 65 active compounds interact with 185 target proteins, as shown in Table 2; in other words, on average, each compound on average interacts with 2.85 target proteins. This result confirms the polypharmacological character of oriental medicine and demonstrates the synergistic effects of multiple compounds on multiple targets [38]. Different compounds in CG and DG can directly affect common targets, for example, the target protein "calmodulin (CALM1)" interacts with crysophanol from CG and coniferyl ferulate from DG at the same time, which implies the synergetic or cumulative effects of herbal medicine.

3.3. GO Analysis. 397 biological process terms with $P$ values of $<0.01$ were sorted using the functional annotation chart of the DAVID 6.8 Gene Functional Classification Tool, based on 185 filtered target genes, and $P$ values were adjusted using the Benjamini-Hochberg method. 30 enriched GO BP terms extracted by $P$ value and gene counts are displayed in Figure 2. It is meaningful that most of the target genes are significantly related to the various BP involved in parturition. For instance, 30 extracted GO BP terms include "MAPK signaling pathways," "steroid hormone mediated signaling pathway," "response to glucocorticoid," "response to estradiol," and "positive regulation of ERK1 and ERK2 cascade." "MAPK signaling pathways" were reported to be activated in human uterine cervical ripening during parturition [39]. "Steroid hormone mediated signaling pathway" is highly related to parturition process as estrogen and progesterone play important roles in pregnancy and parturition, and estrogen induces the principal stimulatory myometrial contractility [40]. Also, estradiol takes key place in parturition process [41]. It was identified that increased ERK activation is observed at the onset of labor, and it promotes myometrial contractility and development of parturition [42, 43]. To sum up, the target genes of BSS are highly associated with the biological process (BP) of parturition.

3.4. Network Construction and Analysis. Network analysis is an efficient tool for visualizing and understanding multiple targeted drug actions and demonstrates drug actions within the context of the whole genome $[44,45]$. For a better insight of therapeutic impacts, $\mathrm{H}-\mathrm{C}-\mathrm{T}$ and T-P networks were constructed and displayed in Figures 3 and 4, respectively. In the H-C-T network, nodes represent herb names, compounds, and targets. Also in the T-P network, circular nodes represent targets and triangle nodes represent pathways. Besides node size is relative to the degree and edges show interactions between nodes.

H-C-T network confirmed that there were 739 interactions between 185 targets and 65 active compounds of CG and DG: oleic acid (C48, degree $=42$ ) with the highest number of interactions with targets, followed by succinic acid $($ C63, degree $=40)$ and stigmasterol (C62, degree $=$ $37)$. It shows that single molecules target multiple receptors [46]. Also, some compounds from CG and DG were found to share common targets. Likewise, prostaglandin $\mathrm{G} / \mathrm{H}$ synthase 2 (PTGS2, degree $=56)$ displayed the most affinitive connections with compounds, followed by gammaaminobutyric acid receptor subunit alpha-1 (GABRA1, degree $=48)$, prostaglandin $\mathrm{G} / \mathrm{H}$ synthase $1(\mathrm{PTGS1}$, degree $=37)$, 
TABLE 2: Related targets of potential compounds in BSS.

\begin{tabular}{|c|c|c|}
\hline UniProt ID & Target name & Gene Name \\
\hline P80404 & 4-aminobutyrate aminotransferase, mitochondrial & ABAT \\
\hline P33121 & Long-chain-fatty-acid--CoA ligase 1 & ACSL1 \\
\hline O60488 & Long-chain-fatty-acid--CoA ligase 4 & ACSL4 \\
\hline P00813 & Adenosine deaminase & $\mathrm{ADA}$ \\
\hline P07327 & Alcohol dehydrogenase $1 \mathrm{~A}$ & $\mathrm{ADH} 1 \mathrm{~A}$ \\
\hline P00325 & Alcohol dehydrogenase $1 \mathrm{~B}$ & $\mathrm{ADH} 1 \mathrm{~B}$ \\
\hline P00326 & Alcohol dehydrogenase $1 \mathrm{C}$ & $\mathrm{ADH} 1 \mathrm{C}$ \\
\hline P29274 & Adenosine A2a receptor & ADORA2A \\
\hline P35348 & Alpha-1A adrenergic receptor & ADRA1A \\
\hline P35368 & Alpha-1B adrenergic receptor & ADRA1B \\
\hline P25100 & Alpha-1D adrenergic receptor & ADRA1D \\
\hline P08913 & Alpha-2A adrenergic receptor & ADRA2A \\
\hline P18089 & Alpha-2B adrenergic receptor & ADRA2B \\
\hline P18825 & Alpha-2C adrenergic receptor & ADRA2C \\
\hline P08588 & Beta-1 adrenergic receptor & ADRB1 \\
\hline P07550 & Beta-2 adrenergic receptor & ADRB2 \\
\hline Q5SY84 & Adenylosuccinate synthetase & ADSS \\
\hline P21549 & Serine--pyruvate aminotransferase & AGXT \\
\hline O43865 & Putative adenosylhomocysteinase 2 & AHCYL1 \\
\hline $\mathrm{P} 15121$ & Aldose reductase & AKR1B1 \\
\hline P13716 & Delta-aminolevulinic acid dehydratase & ALAD \\
\hline P51649 & Succinate semialdehyde dehydrogenase, mitochondrial & ALDH5A1 \\
\hline P04745 & Alpha-amylase 1 & AMY1A \\
\hline P04746 & Pancreatic alpha-amylase & AMY2A \\
\hline P04114 & Apolipoprotein B-100 & APOB \\
\hline P10275 & Androgen receptor & $\mathrm{AR}$ \\
\hline P06576 & ATP synthase subunit beta, mitochondrial & ATP5B \\
\hline P06276 & Cholinesterase & $\mathrm{BCHE}$ \\
\hline P10415 & Apoptosis regulator $\mathrm{Bcl}-2$ & BCL2 \\
\hline Q06187 & Tyrosine-protein kinase BTK & BTK \\
\hline P00915 & Carbonic anhydrase I & CA1 \\
\hline P62158 & Calmodulin & CALM1 \\
\hline P42574 & Caspase-3 & CASP3 \\
\hline P04040 & Catalase & CAT \\
\hline P06307 & Cholecystokinin & CCK \\
\hline P20248 & Cyclin-A2 & CCNA2 \\
\hline P30305 & M-phase inducer phosphatase 2 & CDC25B \\
\hline P24941 & Cell division protein kinase 2 & CDK2 \\
\hline P11597 & Cholesteryl ester transfer protein & CETP \\
\hline P28329 & Choline O-acetyltransferase & CHAT \\
\hline O14757 & Serine/threonine-protein kinase Chk1 & CHEK1 \\
\hline P36222 & Chitinase-3-like protein 1 & CHI3L1 \\
\hline P11229 & Muscarinic acetylcholine receptor M1 & CHRM1 \\
\hline P08172 & Muscarinic acetylcholine receptor M2 & CHRM2 \\
\hline P20309 & Muscarinic acetylcholine receptor M3 & CHRM3 \\
\hline Q15822 & Neuronal acetylcholine receptor subunit alpha-2 & CHRNA2 \\
\hline P36544 & Neuronal acetylcholine receptor protein, alpha-7 chain & CHRNA7 \\
\hline Q99966 & $\mathrm{Cbp} / \mathrm{p} 300$-interacting transactivator 1 & CITED1 \\
\hline P02452 & Collagen alpha-1(I) chain & COL1A1 \\
\hline Q02388 & Collagen alpha-1(VII) chain & COL7A1 \\
\hline P17538 & Chymotrypsinogen B & CTRB1 \\
\hline P07339 & Cathepsin D & CTSD \\
\hline P04798 & Cytochrome P450 1A1 & CYP1A1 \\
\hline
\end{tabular}


TABLE 2: Continued.

\begin{tabular}{|c|c|c|}
\hline UniProt ID & Target name & Gene Name \\
\hline P05177 & Cytochrome P450 1A2 & CYP1A2 \\
\hline Q9ULA0 & Aspartyl aminopeptidase & DNPEP \\
\hline P27487 & Dipeptidyl peptidase IV & DPP4 \\
\hline P21728 & Dopamine D1 receptor & DRD1 \\
\hline P14416 & $\mathrm{D}(2)$ dopamine receptor & DRD2 \\
\hline P25101 & Endothelin-1 & EDNRA \\
\hline Q07075 & Glutamyl aminopeptidase & ENPEP \\
\hline Q6UWV6 & Ectonucleotide pyrophosphatase/phosphodiesterase family member 7 & ENPP7 \\
\hline P04626 & Receptor tyrosine-protein kinase erbB-2 & ERBB2 \\
\hline P03372 & Estrogen receptor & ESR1 \\
\hline Q92731 & Estrogen receptor beta & ESR2 \\
\hline P00742 & Coagulation factor $\mathrm{Xa}$ & F10 \\
\hline P00734 & Thrombin & $\mathrm{F} 2$ \\
\hline P08709 & Coagulation factor VII & F7 \\
\hline P07148 & Fatty acid-binding protein, liver & FABP1 \\
\hline P01100 & Proto-oncogene c-Fos & FOS \\
\hline P15408 & Fos-related antigen 2 & FOSL2 \\
\hline P35575 & Glucose-6-phosphatase & G6PC \\
\hline P14867 & Gamma-aminobutyric acid receptor subunit alpha-1 & GABRA1 \\
\hline P47869 & Gamma-aminobutyric-acid receptor alpha-2 subunit & GABRA2 \\
\hline P34903 & Gamma-aminobutyric-acid receptor alpha-3 subunit & GABRA3 \\
\hline P48169 & Gamma-aminobutyric-acid receptor subunit alpha- 4 & GABRA4 \\
\hline P31644 & Gamma-aminobutyric-acid receptor alpha- 5 subunit & GABRA5 \\
\hline Q16445 & Gamma-aminobutyric-acid receptor subunit alpha- 6 & GABRA6 \\
\hline P17677 & Neuromodulin & GAP43 \\
\hline P47871 & Glucagon & GCGR \\
\hline P14136 & Glial fibrillary acidic protein & GFAP \\
\hline Q2TU84 & Growth-inhibiting protein 18 & GIG18 \\
\hline P23415 & Glycine receptor alpha-1 chain & GLRA1 \\
\hline P00367 & Glutamate dehydrogenase 1, mitochondrial & GLUD1 \\
\hline $\mathrm{P} 17174$ & Aspartate aminotransferase, cytoplasmic & GOT1 \\
\hline P00505 & Aspartate aminotransferase, mitochondrial & GOT2 \\
\hline P42262 & Glutamate receptor 2 & GRIA2 \\
\hline P49841 & Glycogen synthase kinase- 3 beta & GSK3B \\
\hline Q15486 & Putative beta-glucuronidase-like protein SMA3 & GUSBP1 \\
\hline P19367 & Hexokinase-1 & HK1 \\
\hline P04035 & 3-hydroxy-3-methylglutaryl-coenzyme A reductase & HMGCR \\
\hline P00738 & Haptoglobin & HP \\
\hline O14756 & Oxidoreductase & HSD17B6 \\
\hline P08238 & Heat shock protein HSP 90 & HSP90AB1 \\
\hline P28223 & 5-hydroxytryptamine $2 \mathrm{~A}$ receptor & HTR2A \\
\hline P01344 & Insulin-like growth factor II & IGF2 \\
\hline P01857 & Ig gamma-1 chain $\mathrm{C}$ region & IGHG1 \\
\hline P22301 & Interleukin-10 & IL10 \\
\hline P05231 & Interleukin-6 & IL6 \\
\hline P01308 & Insulin & INS \\
\hline Q12809 & Potassium voltage-gated channel subfamily $\mathrm{H}$ member 2 & $\mathrm{KCNH} 2$ \\
\hline Q12791 & Calcium-activated potassium channel subunit alpha 1 & KCNMA1 \\
\hline P35968 & Vascular endothelial growth factor receptor 2 & KDR \\
\hline P09848 & Lactase-phlorizin hydrolase & LCT \\
\hline Q32P28 & Prolyl 3-hydroxylase 1 & LEPRE1 \\
\hline Q8IVL6 & Prolyl 3-hydroxylase 3 & LEPREL2 \\
\hline $\mathrm{P} 06858$ & Lipoprotein lipase & LPL \\
\hline
\end{tabular}


TABLe 2: Continued.

\begin{tabular}{|c|c|c|}
\hline UniProt ID & Target name & Gene Name \\
\hline P09960 & Leukotriene A-4 hydrolase & LTA4H \\
\hline P21397 & Amine oxidase [flavin-containing] A & MAOA \\
\hline P27338 & Amine oxidase [flavin-containing] B & MAOB \\
\hline Q16539 & Mitogen-activated protein kinase 14 & MAPK14 \\
\hline Q00266 & S-adenosylmethionine synthetase isoform type- 1 & MAT1A \\
\hline P31153 & S-adenosylmethionine synthetase isoform type-2 & MAT2A \\
\hline P23368 & NAD-dependent malic enzyme, mitochondrial & ME2 \\
\hline Q16798 & NADP-dependent malic enzyme, mitochondrial & ME3 \\
\hline Q3SYC2 & 2-acylglycerol O-acyltransferase 2 & MOGAT2 \\
\hline P05164 & Myeloperoxidase & MPO \\
\hline Q15788 & Nuclear receptor coactivator 1 & NCOA1 \\
\hline Q15596 & Nuclear receptor coactivator 2 & NCOA2 \\
\hline P29475 & Nitric-oxide synthase, brain & NOS1 \\
\hline P35228 & Nitric oxide synthase, inducible & NOS2 \\
\hline P29474 & Nitric oxide synthase, endothelial & NOS3 \\
\hline P16083 & NRH dehydrogenase [quinone] 2 & NQO2 \\
\hline Q14994 & Nuclear receptor subfamily 1 group I member 3 & NR1I3 \\
\hline P04150 & Glucocorticoid receptor & $\mathrm{NR} 3 \mathrm{C} 1$ \\
\hline P08235 & Mineralocorticoid receptor & NR3C2 \\
\hline Q16620 & BDNF/NT-3 growth factors receptor & NTRK2 \\
\hline P04181 & Ornithine aminotransferase, mitochondrial & OAT \\
\hline P00480 & Ornithine carbamoyltransferase, mitochondrial & OTC \\
\hline Q9BYC2 & Succinyl-CoA:3-ketoacid-coenzyme A transferase 2, mitochondrial & OXCT2 \\
\hline O15460 & Prolyl 4-hydroxylase subunit alpha-2 & P4HA2 \\
\hline P49585 & Choline-phosphate cytidylyltransferase A & PCYT1A \\
\hline Q14432 & CGMP-inhibited $3^{\prime}, 5^{\prime}$-cyclic phosphodiesterase A & PDE3A \\
\hline O00330 & Pyruvate dehydrogenase protein X component, mitochondrial & PDHX \\
\hline P52945 & Pancreas/duodenum homeobox protein 1 & PDX1 \\
\hline P06401 & Progesterone receptor & PGR \\
\hline P48736 & Phosphatidylinositol-4,5-bisphosphate 3-kinase catalytic subunit, gamma isoform & PIK3CG \\
\hline P11309 & Proto-oncogene serine/threonine-protein kinase Pim-1 & PIM1 \\
\hline P61925 & cAMP-dependent protein kinase inhibitor alpha & PKIA \\
\hline P14618 & Pyruvate kinase isozymes M1/M2 & PKM2 \\
\hline P04054 & Phospholipase A2 & PLA2G1B \\
\hline P00749 & Urokinase-type plasminogen activator & PLAU \\
\hline P00747 & Plasminogen & PLG \\
\hline P00491 & Purine nucleoside phosphorylase & PNP \\
\hline P27169 & Serum paraoxonase/arylesterase 1 & PON1 \\
\hline Q07869 & Peroxisome proliferator-activated receptor alpha & PPARA \\
\hline Q03181 & Peroxisome proliferator-activated receptor delta & PPARD \\
\hline P37231 & Peroxisome proliferator activated receptor gamma & PPARG \\
\hline Q9UBK2 & Peroxisome proliferator-activated receptor gamma coactivator 1-alpha & PPARGC1A \\
\hline P17612 & mRNA of PKA Catalytic Subunit C-alpha & PRKACA \\
\hline P05771 & Protein kinase $\mathrm{C}$ beta type & PRKCB \\
\hline P35030 & Trypsin-3 & PRSS3 \\
\hline P60484 & Phosphatidylinositol-3,4,5-trisphosphate 3-phosphatase and dual-specificity protein phosphatase PTEN & PTEN \\
\hline P43115 & Prostaglandin E2 receptor EP3 subtype & PTGER3 \\
\hline P23219 & Prostaglandin G/H synthase 1 & PTGS1 \\
\hline P35354 & Prostaglandin G/H synthase 2 & PTGS2 \\
\hline P18031 & mRNA of Protein-tyrosine phosphatase, non-receptor type 1 & PTPN1 \\
\hline P10082 & Peptide YY & PYY \\
\hline P63000 & Ras-related C 3 botulinum toxin substrate 1 & RAC1 \\
\hline P50120 & Retinol-binding protein 2 & RBP2 \\
\hline P08100 & Rhodopsin & $\mathrm{RHO}$ \\
\hline
\end{tabular}


TABLE 2: Continued.

\begin{tabular}{|c|c|c|}
\hline UniProt ID & Target name & Gene Name \\
\hline P19793 & Retinoic acid receptor RXR-alpha & RXRA \\
\hline O00767 & Acyl-CoA desaturase & SCD \\
\hline Q14524 & Sodium channel protein type 5 subunit alpha & SCN5A \\
\hline P31040 & Succinate dehydrogenase [ubiquinone] flavoprotein subunit, mitochondrial & SDHA \\
\hline P16109 & P-selectin & SELP \\
\hline P05121 & Plasminogen activator inhibitor 1 & SERPINE1 \\
\hline P14410 & Sucrase-isomaltase, intestinal & SI \\
\hline O76082 & Solute carrier family 22 member 5 & SLC22A5 \\
\hline Q9UBX3 & Mitochondrial dicarboxylate carrier & SLC25A10 \\
\hline P11168 & Solute carrier family 2 , facilitated glucose transporter member 2 & SLC2A2 \\
\hline P23975 & Sodium-dependent noradrenaline transporter & SLC6A2 \\
\hline Q01959 & Sodium-dependent dopamine transporter & SLC6A3 \\
\hline P31645 & Sodium-dependent serotonin transporter & SLC6A4 \\
\hline P35610 & Sterol O-acyltransferase 1 & SOAT1 \\
\hline P00441 & Superoxide dismutase $[\mathrm{Cu}-\mathrm{Zn}]$ & SOD1 \\
\hline P08047 & Transcription factor Sp1 & SP1 \\
\hline P12931 & Proto-oncogene tyrosine-protein kinase SRC & SRC \\
\hline P36956 & Sterol regulatory element-binding protein 1 & SREBF1 \\
\hline Q12772 & Sterol regulatory element-binding protein 2 & SREBF2 \\
\hline Q9P2R7 & Succinyl-CoA ligase [ADP-forming] beta-chain, mitochondrial & SUCLA2 \\
\hline Q99973 & Telomerase protein component 1 & TEP1 \\
\hline P01375 & Tumor necrosis factor & TNF \\
\hline Q16881 & Thioredoxin reductase, cytoplasmic & TXNRD1 \\
\hline P55851 & Mitochondrial uncoupling protein 2 & UCP2 \\
\hline P55916 & Mitochondrial uncoupling protein 3 & UCP3 \\
\hline
\end{tabular}

and muscarinic acetylcholine receptor M1 (CHRM1, degree = 37). Except for C60 (PLA2G1B, degree = 1), the rest of the 64 active compounds are connected with more than one target; likewise, 73 (39.5\%) target genes out of 185 interacted with more than one compound. This result demonstrates the multicompounds and multitarget properties of herbal compounds and there was a report that compounds with multiple targets could have greater therapeutic efficacy [47].

In addition, the top 40 pathways were extracted based on gene counts and $P$ value $(<0.05)$, and $P$ value was adjusted by Benjamini-Hochberg method. T-P network using relevant targets of herbal compounds is demonstrated in Figure 4. There were 485 interactions between the top 40 pathways and 135 of 185 target genes. "Metabolic pathways" (degree $=49$ ) and "neuroactive ligand-receptor interaction pathway" (degree = 32) had the highest and the second highest numbers of connections with the targets, followed by "calcium signaling" (degree = 21), "cAMP signaling pathway" (degree $=17$ ), and "cGMP PKG signaling pathway" (degree $=15)$. These are compelling results that parturition processes are the complex hormone interactions and it is well known that calcium signals within the myometrium are pivotal for uterine contractions [48]. In the same manner, some target genes demonstrated higher degree centrality with top 40 pathways, namely, PI3-kinase subunit gamma (PIK3CG, degree $=23$, , AMP-dependent protein kinase catalytic subunit alpha (PRKACA, degree $=20$ ), protein kinase $C$ beta type $(P R K C B$, degree $=18)$, and calmodulin (CALM1, degree $=11$ ). We can confirm the same result in the previous researches. For instance, PI3-kinase subunit gamma plays the key role in regulating cAMP, calcium cycling, and beta-adrenergic signaling [49]. Moreover, during the labor, calmodulin-calcium complex activates myosin lightchain kinase, which causes the generation of ATPase activity; eventually, uterine contraction is promoted [50].

H-C-T network explains the multitarget, multicompounds properties and accumulates effect of herbal medicines and T-P network shows that target genes of BSS are highly related to the pathway associated with parturition process.

3.5. Target Organ Location Map. It is important to confirm the tissue mRNA expression profiles of the target genes at the organ level to identify the effects of BSS on parturition. Since there was no mRNA expression information in BioGPS of muscarinic acetylcholine receptor M1 (CHRM1), putative beta-glucuronidase-like protein SMA3 (GUSBP1), and retinol-binding protein 2 (RBP2), excluding these 3 targets from 185 filtered targets, totally 182 genes mRNA expression profiles were analyzed in this study. There were 519 interactions between target genes and organ locations. The networks of target genes tissue mRNA expression profiles and compounds of BSS are shown in Figure 5. 


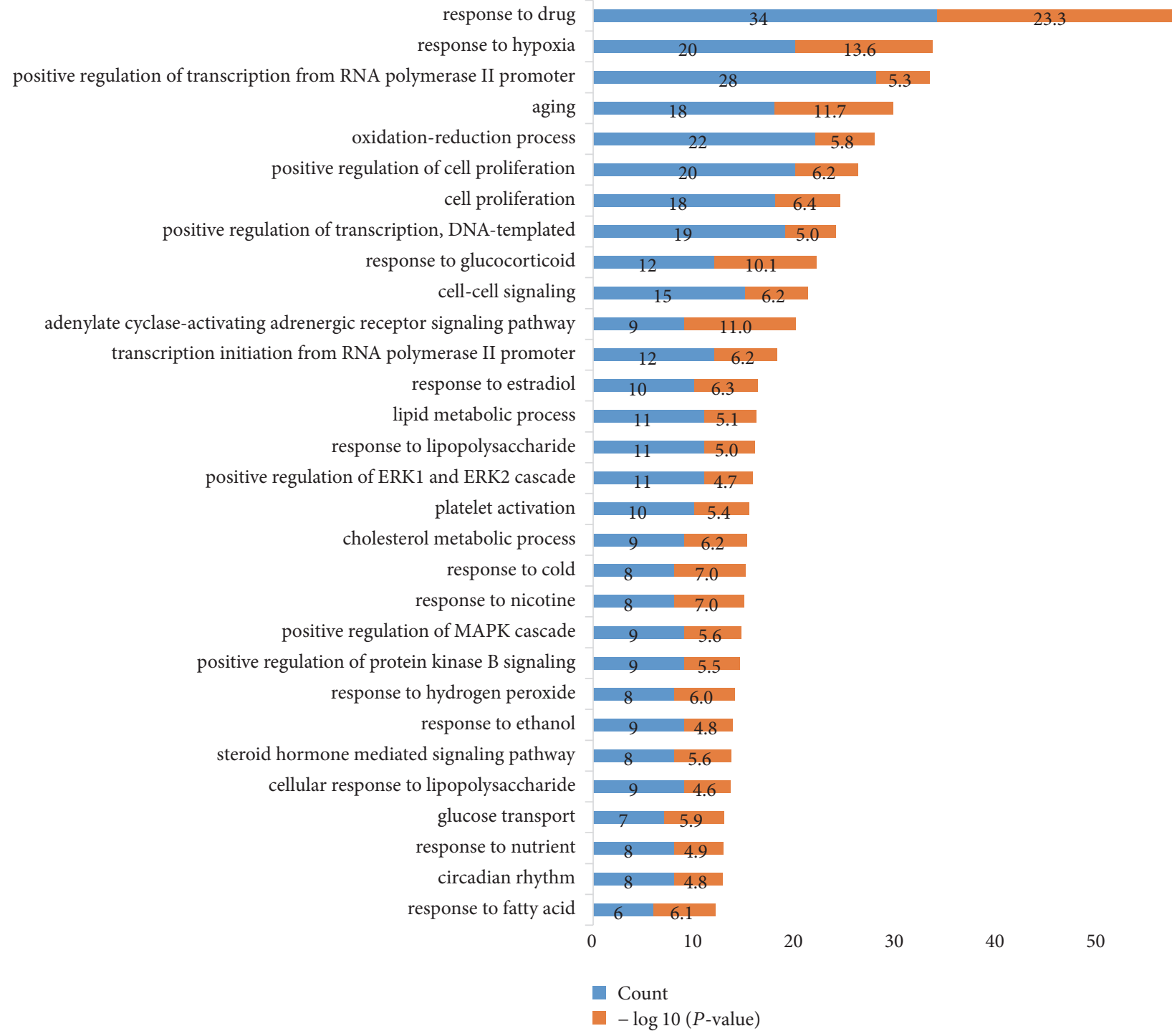

FIGURE 2: GO analysis: 30 enriched biological process (BP) of gene ontology (GO) terms sorted by $P$ value $<0.01$ and gene counts are displayed. The $y$-axis represents enriched biological process (BP) terms for the target genes, and the $x$-axis shows gene counts and $-\log 10(P$ value).

As a result, 159 of 182 target genes displayed beyond average mRNA expression in relevant organ tissues, such as uterus and/or uterus corpus, fetus and/or placenta, hypothalamus and/or pituitary, smooth muscle, and whole blood. The rest of 23 genes of 182 targets did not display above average mRNA expression in above organ tissues, for example, gammaaminobutyric acid receptor subunit alpha-6 (GABRA6) and coagulation factor X (F10).

Nevertheless, most genes of 159 demonstrated high expression patterns in several organs of parturition related tissues at the same time. In detail, 60 genes showed most significant mRNA expression in the uterus and/or uterus corpus group, 130 for placenta and/or fetus, 86 for hypothalamus and/or pituitary, 82 for smooth muscle, 80 for pituitary, and 81 for whole blood. Besides, 30 of 159 genes showed expression in all of 6 groups. For instance, muscarinic acetylcholine receptor M2 (CHRM2), neuronal acetylcholine receptor subunit $\alpha$-2 (CHRNA2), gamma-aminobutyric acid receptor subunit alpha-3 (GABRA3), NO synthase, inducible (NOS2), cGMP-inhibited $3^{\prime}, 5^{\prime}$-cyclic phosphodiesterase A (PDE3A), and sodium-dependent dopamine transporter (SLC6A3) recorded beyond average mRNA expression in all six groups. Furthermore, 79\% of targets were expressed in two or more organ tissues, which suggests that those organs and target genes of BSS are closely correlated.

\section{Discussion}

In this study, network pharmacology method with DL, OB, Caco-2, and LR evaluation, multiple drug-target prediction, 


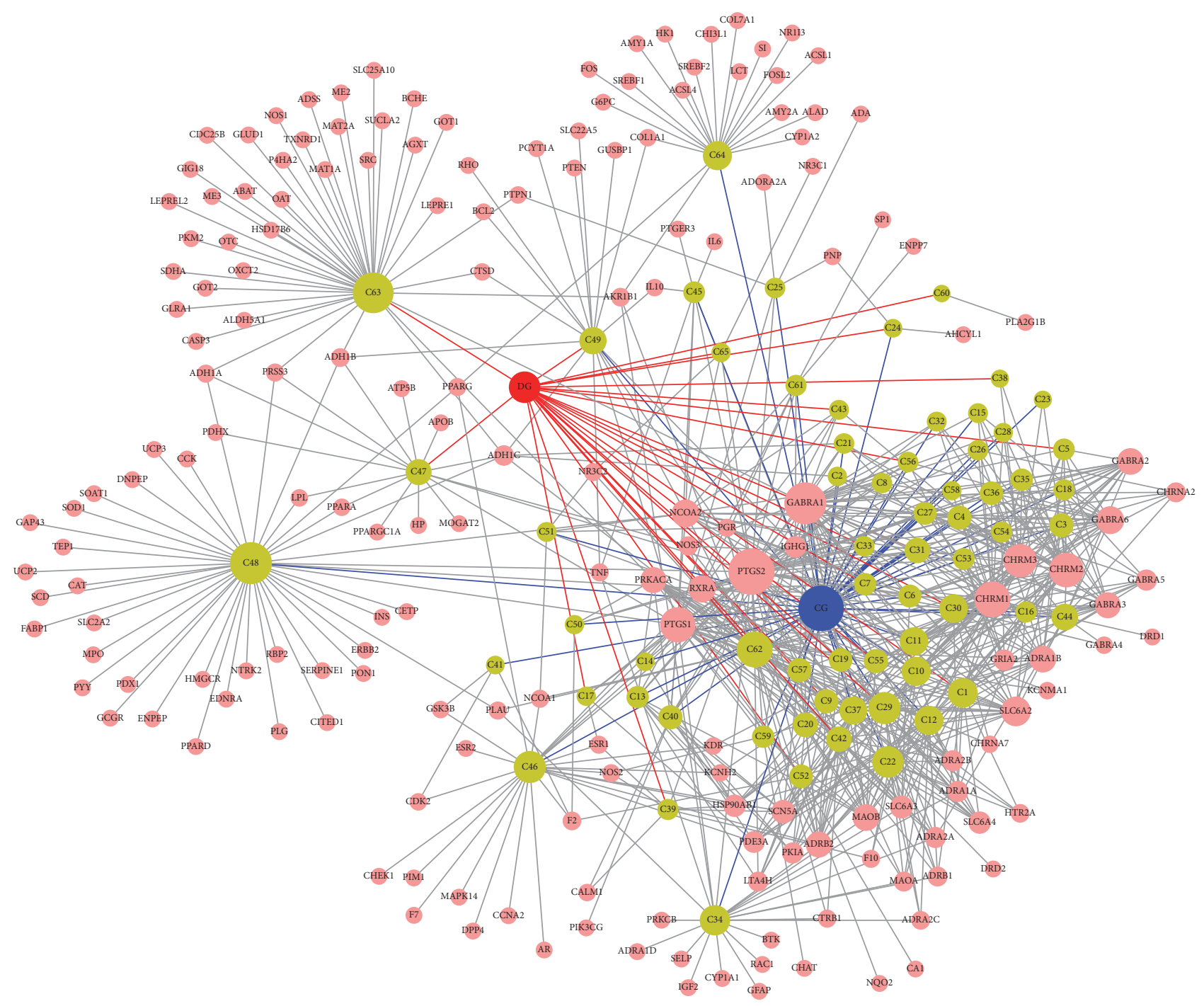

FIGURE 3: H-C-T network: herb-compound-target (H-C-T) network demonstrated multicompound, multitarget property of BSS. In this network, red and blue nodes represent herbs, green nodes show compounds, and pink nodes indicate targets and node size is relative to the degree and edges demonstrate interactions between nodes.

network analysis, and relevant organ location mapping was used to explain the targets of BSS in relation to the parturition process. There is no denying that network based analysis is powerful approach for identifying the actions of multitargeting herbal medicines at the systems level and our study shows target genes of BSS are strongly connected to parturition related pathways, biological processes, and organs. It was confirmed that $98 \%$ of the active compounds of BSS were interacted with more than two targets and 39.5\% of the targets related to more than one compound. The synergetic multitarget properties of BSS were visualized, but further discussion about differentiated drug action based on degree centrality and simultaneous targeting effect of more than one compound is required [51]. Also, detailed potential pathways of BSS should be explored deeply in the future.

Similar findings were identified in a few RCT researches in China that using BSS in induction of labor can reduce the delivery time, the amount of bleeding, and the residual rate of placenta $[52,53]$. In addition, BSS targets six genes of GABA receptor and NOS, which was reported to be related oxytocin neurons at the time of parturition in rats [54]. Also, BSS targets NOS and NO (nitric oxide) which are involved in the regulation of uterine contractility during pregnancy and is a key factor for the onset of labor [55], and iNOS (inducible nitric oxide synthase) can be upregulated accordantly by similar inflammatory mediators during ripening [11].

In fact, rather than DG, Angelicae Gigantis Radix (Danggwi, AGR) grows naturally in Korea; for that reason, the combination of AGR and CG is commonly used as BSS in Korea. Instead, DG is named as Chinese Danggwi for accurate classification in Korea. Several studies have shown AGR is differs from DG in terms of its main active constituents and genetic form. AGR is mainly composed of 


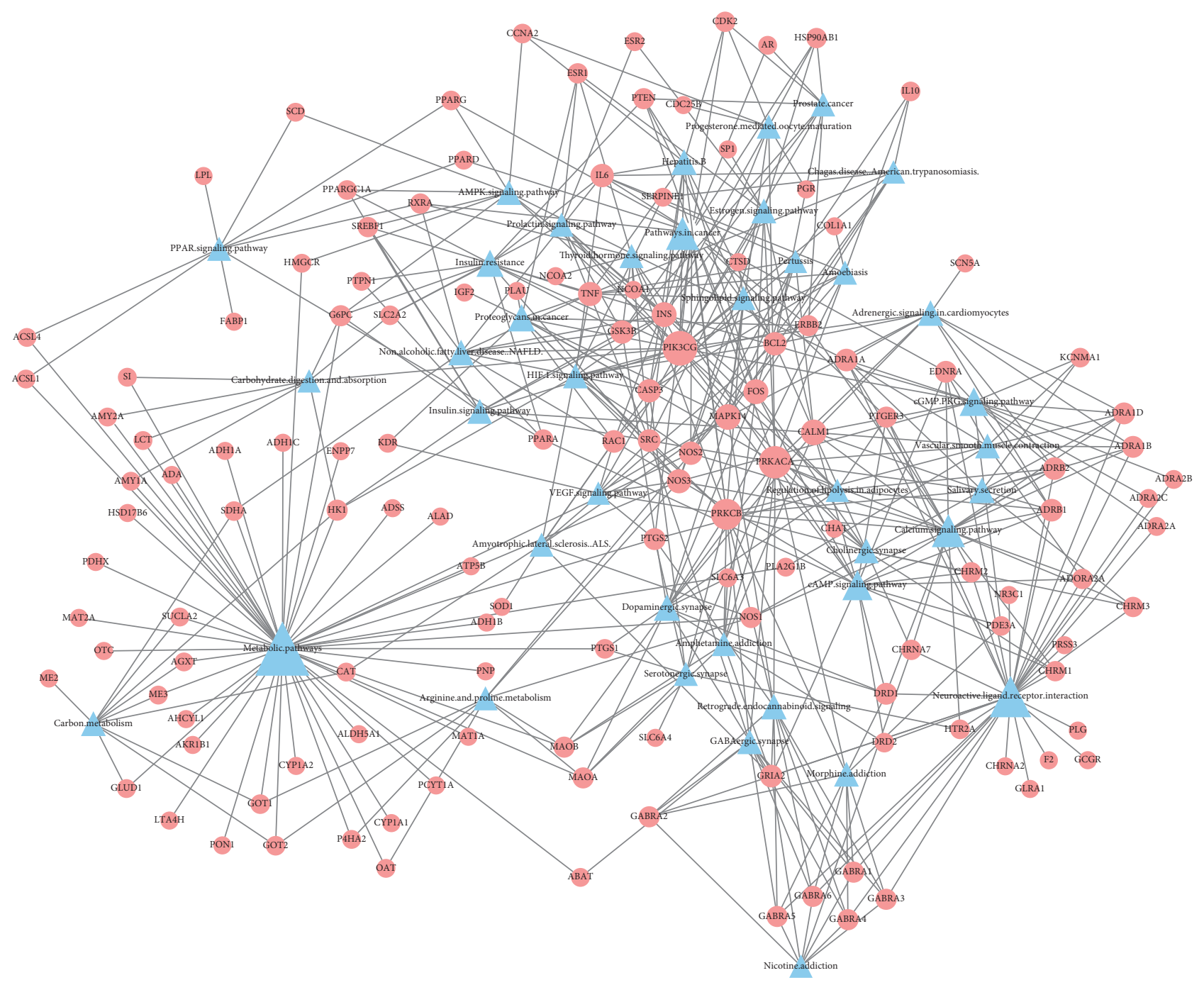

FIGURE 4: T-P network: in target-pathway (T-P) network, circular nodes represent compounds and triangles indicate pathways. Node size is relative to the degree and edges demonstrate interactions between nodes.

water soluble polysaccharide but coumarin, which is liposoluble including nodakenin (1), peucedanone (2), marmesin (3), decursinol (4), 7-hydroxy-6-(2R-hydroxy-3-methylbut3-enyl) coumarin (5), demethylsuberosin (6), decursin (7), decursinol angelate (8), and isoimperatorin (9) [56]. Of these, decursin and its isomer decursinol angelate have been reported to be the active compounds in AGR [57]. It was identified in the experimental studies that AGR and DG act via different mechanisms in the cardiovascular, central nervous system, and anticancer activity but both have similar pharmacological effects [57]. Since the compositions of DG and AGR differ, further study on BSS with AGR is required. Currently, BSS is commonly prescribed to treat cerebra vascular and cardiovascular diseases in China [33], but, in Korea, BSS is widely applied in obstetrics.

The similarity between cervical ripening during parturition and inflammatory reaction has been pointed out in earlier studies; this has been attributed to the induction of leukocyte migration into tissue, thus promoting cervical remodeling and parturition by estrogen [58]. Further study is needed in terms of the effect of BSS on inflammatory reactions and parturition.

Furthermore, the CG-DG herb pair has other names, such as, Gunggui-tang (weight ratios of $2: 3$ or 1:1), Ogeum-san (1:1), Iphyo-san (1:1), and Sinmyo Bulsu-san (1:2), those are prepared at different weight ratios [3]. Accordingly, weight ratio should be determined based on considerations of targeted symptoms for relevant clinical applications.

\section{Conclusion}

This study results show that Bulsu-san (BSS) is highly connected to the parturition related pathways, biological processes, and organs. Most compounds in BSS work together with multiple target genes in a synergetic way, and this was confirmed using herb-compound-target network and targetpathway network analysis. The mRNA expression of relevant target genes of BSS was elevated significantly in parturition 


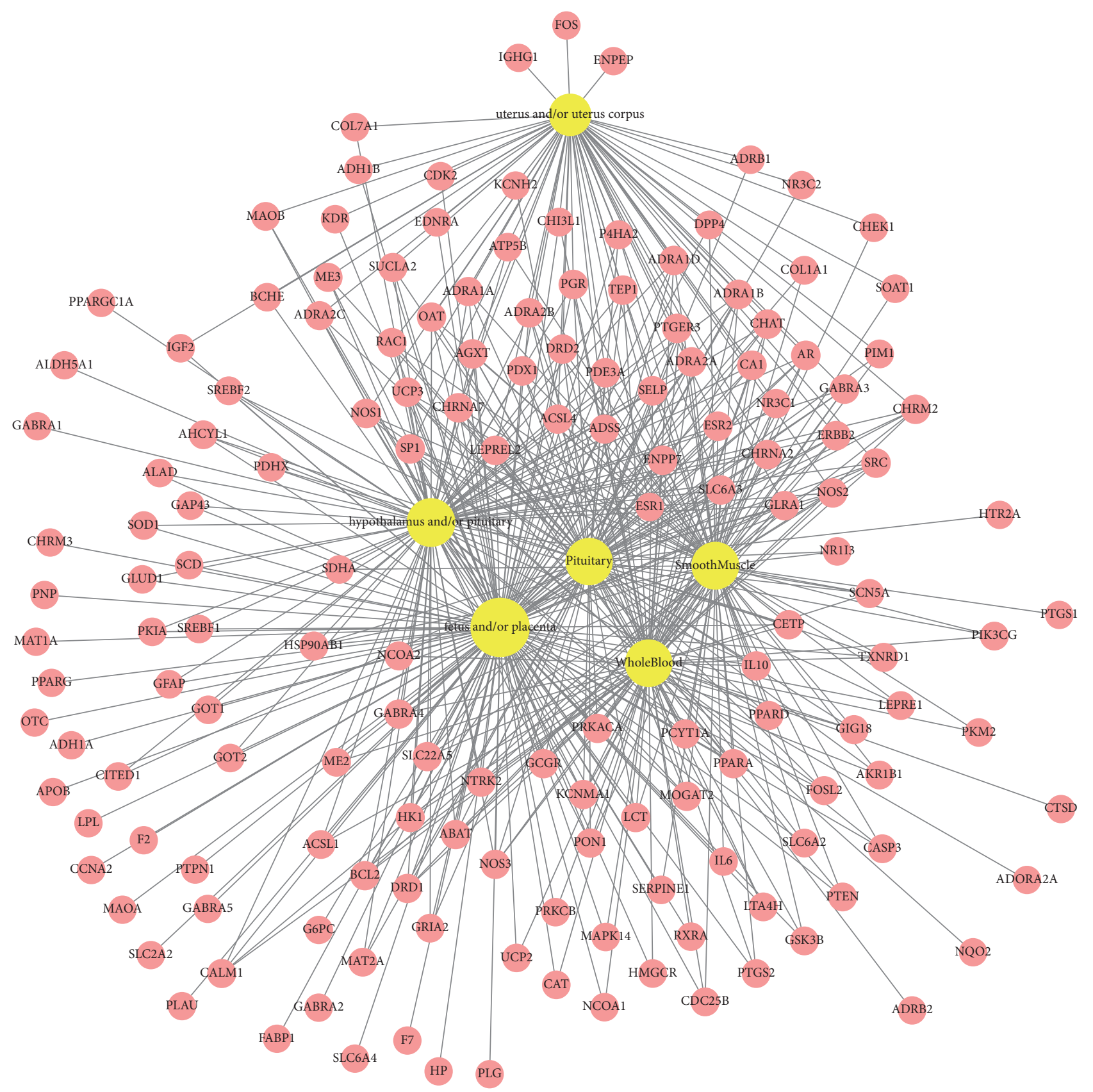

FIGURE 5: Target organ location map: it shows that tissue-specific patterns of mRNA expression are highly active in relative organs of parturition process such as uterus, fetus, placenta, hypothalamus, pituitary, and smooth muscle. Yellow nodes show compounds and pink nodes indicate targets and node size is relative to the degree and edges demonstrate interactions between nodes.

related organ tissues, such as those of the uterus, placenta, fetus, hypothalamus, and pituitary gland.

This study employed the network analytical methods to show the multicompound, multitarget properties of BSS. The results not only support clinical applications of BSS on easing childbirth but also suggest the related target genes and pathways of BSS on promoting parturition according to a systems-level in silico analytic approach. However, detailed mechanisms and other functions of BSS should be discussed further.

\section{Conflicts of Interest}

The authors declare that there are no conflicts of interest.

\section{Acknowledgments}

This research was supported by Basic Science Research Program through the National Research Foundation of Korea (NRF) funded by the Ministry of Education (NRF2015R1D1A1A01059994). 


\section{References}

[1] S. Kim and Y. Lee, "The prescriptions of enriching blood and nourishing vital essence (Fill yin blood prescription)," Journal of Korean Medical Classics, vol. 20, pp. 67-77, 2007.

[2] Y. Jin, C. Qu, Y. Tang et al., "Herb pairs containing Angelicae Sinensis Radix (Danggui): a review of bio-active constituents and compatibility effects," Journal of Ethnopharmacology, vol. 181, pp. 158-171, 2016.

[3] J. Lyu and C. Jeong, "Constitution of prescription and medicinal effect adaptation diseases of 'bullsoosan (berghean)'in korean medical books," Journal of Korean Medical classics, vol. 29, pp. 17-41, 2016.

[4] W. Li, Y. Tang, J. Guo et al., "Enriching blood effect comparison in three kinds of blood deficiency model after oral administration of drug pair of angelicae sinensis radix and chuanxiong rhizoma and each single herb," China Journal of Chinese Materia Medica, vol. 36, no. 13, pp. 1808-1814, 2011.

[5] W. Li, M. Huang, Y. Tang, J. Guo, E. Shang, and X. Liu, "Establishment and optimization of acute blood stasis rat model," Chinese Pharmacological Bulletin, vol. 12, p. 032, 2011.

[6] Y.-Z. Hou, G.-R. Zhao, Y.-J. Yuan, G.-G. Zhu, and R. Hiltunen, "Inhibition of rat vascular smooth muscle cell proliferation by extract of Ligusticum chuanxiong and Angelica sinensis," Journal of Ethnopharmacology, vol. 100, no. 1-2, pp. 140-144, 2005.

[7] C. W. C. Bi, L. Xu, X. Y. Tian et al., "Fo Shou San, an ancient chinese herbal decoction, protects endothelial function through increasing endothelial nitric oxide synthase activity," PLoS ONE, vol. 7, no. 12, Article ID e51670, 2012.

[8] W. Li, Y. Hua, Y. Tang, H. Wang, L. Qian, and M. Gao, "Effects of radix angelicae sinensis and rhizoma chuanxiong on mouse uterine contractions in vitro," Journal of Nanjing University of Traditional Chinese Medicine, vol. 2, p. 014, 2010.

[9] M. McLean and R. Smith, "Corticotrophin-releasing hormone and human parturition," Reproduction, vol. 121, no. 4, pp. 493501, 2001.

[10] S. K. Kota, K. Gayatri, S. Jammula et al., "Endocrinology of parturition," Indian Journal of Endocrinology and Metabolism, vol. 17, no. 1, pp. 50-59, 2013.

[11] M. Ledingham, A. J. Thomson, I. A. Greer, and J. E. Norman, "Nitric oxide in parturition," BJOG: An International Journal of Obstetrics \& Gynaecology, vol. 107, pp. 581-593, 2000.

[12] M. Shen, S. Tian, Y. Li et al., "Drug-likeness analysis of traditional Chinese medicines: 1 . property distributions of druglike compounds, non-drug-like compounds and natural compounds from traditional Chinese medicines," Journal of Cheminformatics, vol. 4, no. 1, article 31, 2012.

[13] X. Xu, W. Zhang, C. Huang et al., "A novel chemometric method for the prediction of human oral bioavailability," International Journal of Molecular Sciences, vol. 13, no. 6, pp. 6964-6982, 2012.

[14] I. Hubatsch, E. G. E. Ragnarsson, and P. Artursson, "Determination of drug permeability and prediction of drug absorption in Caco-2 monolayers," Nature Protocols, vol. 2, no. 9, pp. 21112119, 2007.

[15] W. Tao, X. Xu, X. Wang et al., "Network pharmacology-based prediction of the active ingredients and potential targets of Chinese herbal Radix Curcumae formula for application to cardiovascular disease," Journal of Ethnopharmacology, vol. 145, no. 1, pp. 1-10, 2013.

[16] J. Zhang, Y. Li, S.-S. Chen et al., "Systems pharmacology dissection of the anti-inflammatory mechanism for the medicinal herb Folium eriobotryae," International Journal of Molecular Sciences, vol. 16, no. 2, pp. 2913-2941, 2015.

[17] P. Willett, J. M. Barnard, and G. M. Downs, "Chemical similarity searching," Journal of Chemical Information and Computer Sciences, vol. 38, no. 6, pp. 983-996, 1998.

[18] H. Liu, J. Wang, W. Zhou, Y. Wang, and L. Yang, "Systems approaches and polypharmacology for drug discovery from herbal medicines: an example using licorice," Journal of Ethnopharmacology, vol. 146, no. 3, pp. 773-793, 2013.

[19] K. S. Pang, "Modeling of intestinal drug absorption: roles of transporters and metabolic enzymes (for the gillette review series)," Drug Metabolism \& Disposition, vol. 31, no. 12, pp. 15071519, 2003.

[20] T. Pei, C. Zheng, C. Huang et al., "Systematic understanding the mechanisms of vitiligo pathogenesis and its treatment by Qubaibabuqi formula," Journal of Ethnopharmacology, vol. 190, pp. 272-287, 2016.

[21] C. A. Lipinski, F. Lambardo, B. W. Dominy, and P. J. Feeney, "Experimental and computational approaches to estimate solubility and permeability in drug discovery and development settings," Advanced Drug Delivery Reviews, vol. 64, pp. 4-17, 2012.

[22] A. K. Ghose, V. N. Viswanadhan, and J. J. Wendoloski, "Prediction of hydrophobic (lipophilic) properties of small organic molecules using fragmental methods: an analysis of ALOGP and CLOGP methods," The Journal of Physical Chemistry A, vol. 102, no. 21, pp. 3762-3772, 1998.

[23] P. Rajasethupathy, S. J. Vayttaden, and U. S. Bhalla, "Systems modeling: a pathway to drug discovery," Current Opinion in Chemical Biology, vol. 9, no. 4, pp. 400-406, 2005.

[24] H. Yu, J. Chen, X. Xu et al., "A systematic prediction of multiple drug-target interactions from chemical, genomic, and pharmacological data," PLoS ONE, vol. 7, no. 5, Article ID e37608, 2012.

[25] C. H. Wu, R. Apweiler, A. Bairoch, D. A. Natale, W. C. Barker, B. Boeckmann et al., "The universal protein resource (UniProt): an expanding universe of protein information," Nucleic Acids Research, vol. 34, pp. D187-D191, 2006.

[26] G. Bindea, B. Mlecnik, H. Hackl et al., "ClueGO: a Cytoscape plug-in to decipher functionally grouped gene ontology and pathway annotation networks," Bioinformatics, vol. 25, no. 8, pp. 1091-1093, 2009.

[27] M. E. Smoot, K. Ono, J. Ruscheinski, P. L. Wang, and T. Ideker, "Cytoscape 2.8: new features for data integration and network visualization," Bioinformatics, vol. 27, no. 3, pp. 431-432, 2011.

[28] J.-B. Pan, S.-C. Hu, D. Shi et al., "PaGenBase: a pattern gene database for the global and dynamic understanding of gene function," PLoS ONE, vol. 8, no. 12, Article ID e80747, 2013.

[29] W. Zhang, Q. Tao, Z. Guo et al., "Systems pharmacology dissection of the integrated treatment for cardiovascular and gastrointestinal disorders by traditional chinese medicine," Scientific Reports, vol. 6, p. 32400, 2016.

[30] Z. Prevoršek, G. Gorjanc, B. Paigen, and S. Horvat, "Congenic and bioinformatics analyses resolved a major-effect Fob3b QTL on mouse Chr 15 into two closely linked loci," Mammalian Genome, vol. 21, no. 3-4, pp. 172-185, 2010.

[31] J. Du, B. Bai, X. Kuang et al., "Ligustilide inhibits spontaneous and agonists- or $\mathrm{K}^{+}$depolarization-induced contraction of rat uterus," Journal of Ethnopharmacology, vol. 108, no. 1, pp. 54$58,2006$. 
[32] K. J. Chen and K. Chen, "Ischemic stroke treated with ligusticum chuanxiong," Chinese Medical Journal, vol. 105, pp. 870873, 1992.

[33] W. Li, J. Guo, Y. Tang et al., "Pharmacokinetic comparison of ferulic acid in normal and blood deficiency rats after oral administration of angelica sinensis, ligusticum chuanxiong and their combination," International Journal of Molecular Sciences, vol. 13, no. 3, pp. 3583-3597, 2012.

[34] W. Karl, W. Cathy, X. Li, and G. Amy, "Pharmacokinetic analyses of ferulic acid in rat plasma by liquid chromatography?tandem mass spectrometry: a synergistic action of an ancient herbal decoction fo shou san," Pharmaceutica Analytica Acta, vol. 6, no. 5, p. 2, 2015.

[35] D. Huang, "8 purgative herbs," Chinese Materia Medica: Chemistry, Pharmacology and Applications, p. 231, 1998.

[36] X. Liu, Q. Zhao, C. Li, R. Zhang, X. Wang, and W. Xu, "Synthesis of chuanxiong perlolyrine and its protective effect on injured vascular endothelial cells," Journal of Shandong University (Health Sciences), vol. 41, pp. 485-487, 2003.

[37] J. Ishida, M. Kozuka, H.-K. Wang et al., "Antitumor-promoting effects of cyclic diarylheptanoids on Epstein-Barr virus activation and two-stage mouse skin carcinogenesis," Cancer Letters, vol. 159, no. 2, pp. 135-140, 2000.

[38] J. Liu, T. Pei, and J. Mu, "Systems pharmacology uncovers the multiple mechanisms of Xijiao Dihuang decoction for the treatment of viral hemorrhagic fever," Evidence-Based Complementary and Alternative Medicine, vol. 2016, Article ID 9025036, 17 pages, 2016.

[39] H. Wang and Y. V. Stjernholm, "Plasma membrane receptor mediated MAPK signaling pathways are activated in human uterine cervix at parturition," Reproductive Biology and Endocrinology, vol. 5, article 3, p. 1, 2007.

[40] S. Mesiano and T. N. Welsh, "Steroid hormone control of myometrial contractility and parturition," Seminars in Cell \& Developmental Biology, vol. 18, no. 3, pp. 321-331, 2007.

[41] P. W. Nathanielsz, S. L. Jenkins, J. D. Tame, J. A. Winter, S. Guller, and D. A. Giussani, "Local paracrine effects of estradiol are central to parturition in the rhesus monkey," Nature Medicine, vol. 4, no. 4, pp. 456-459, 1998.

[42] Y. Li, H. Je, S. Malek, and K. G. Morgan, "ERK1/2-mediated phosphorylation of myometrial caldesmon during pregnancy and labor," American Journal of Physiology-Regulatory, Integrative and Comparative Physiology, vol. 284, no. 1, pp. R192-R199, 2003.

[43] Y. Li, H.-D. Je, S. Malek, and K. G. Morgan, "Role of ERK1/2 in uterine contractility and preterm labor in rats," American Journal of Physiology-Regulatory, Integrative and Comparative Physiology, vol. 287, no. 2, pp. R328-R335, 2004.

[44] S. I. Berger and R. Iyengar, "Network analyses in systems pharmacology," Bioinformatics, vol. 25, no. 19, pp. 2466-2472, 2009.

[45] P. Li, L.-W. Qi, E.-H. Liu, J.-L. Zhou, and X.-D. Wen, “Analysis of Chinese herbal medicines with holistic approaches and integrated evaluation models," TrAC Trends in Analytical Chemistry, vol. 27, no. 1, pp. 66-77, 2008.

[46] L. M. Espinoza-Fonseca, "The benefits of the multi-target approach in drug design and discovery," Bioorganic \& Medicinal Chemistry, vol. 14, no. 4, pp. 896-897, 2006.

[47] W. Zhang, Y. Bai, Y. Wang, and W. Xiao, "Polypharmacology in drug discovery: a review from systems pharmacology perspective," Current Pharmaceutical Design, vol. 22, no. 21, pp. 31713181, 2016.
[48] S. Wray, K. Jones, and S. Kupittayanant, "Calcium signaling and uterine contractility," Journal of the Society for Gynecologic Investigation, vol. 10, no. 5, pp. 252-264, 2003.

[49] G. Y. Oudit and Z. Kassiri, "Role of PI3 kinase gamma in excitation-contraction coupling and heart disease," Cardiovascular and Hematological Disorders, vol. 7, no. 4, pp. 295-304, 2007.

[50] R. Smith, "Parturition," The New England Journal of Medicine, vol. 356, no. 3, pp. 271-283, 2007.

[51] S. Y. Suh and W. G. An, "Systems pharmacological approach of pulsatillae radix on treating crohn's disease," Evidence-Based Complementary and Alternative Medicine, vol. 2017, Article ID 4198035, 21 pages, 2017.

[52] C. 1. Chen and J. Y. Chen, "Analysis on the effect of foshousan combined with ethacridine lactate in mid-term pregnancy," Journal of Practical Traditional Chinese Medicine, vol. 30, pp. 511512, 2014.

[53] Y. Sun, "Modified Xiong Gui Tang with auricular acupoint press for 70 cases of late pregnancy," Guide of China Medicine, vol. 7, no. 20, pp. 92-93, 2009.

[54] J.-J. Koksma, J.-M. Fritschy, V. MacK, R. E. Van Kesteren, and A. B. Brussaard, "Differential GABA A receptor clustering determines GABA synapse plasticity in rat oxytocin neurons around parturition and the onset of lactation," Molecular and Cellular Neuroscience, vol. 28, no. 1, pp. 128-140, 2005.

[55] H. Maul, M. Longo, G. R. Saade, and R. E. Garfield, "Nitric oxide and its role during pregnancy: From ovulation to delivery," Current Pharmaceutical Design, vol. 9, no. 5, pp. 359-380, 2003.

[56] M.-J. Ahn, M. K. Lee, Y. C. Kim, and S. H. Sung, "The simultaneous determination of coumarins in Angelica gigas root by high performance liquid chromatography-diode array detector coupled with electrospray ionization/mass spectrometry," Journal of Pharmaceutical and Biomedical Analysis, vol. 46, no. 2, pp. 258-266, 2008.

[57] S. Kim, H. Oh, J. Kim, J. Hong, and S. Cho, "A review of pharmacological effects of angelica gigas, angelica sinensis, angelica acutiloba and their bioactive compounds," The Journal of Korean Oriental Medicine, vol. 32, pp. 1-24, 2011.

[58] J. E. Norman, S. Bollapragada, M. Yuan, and S. M. Nelson, "Inflammatory pathways in the mechanism of parturition," BMC Pregnancy and Childbirth, vol. 7, supplement 1, article S7, 2007. 


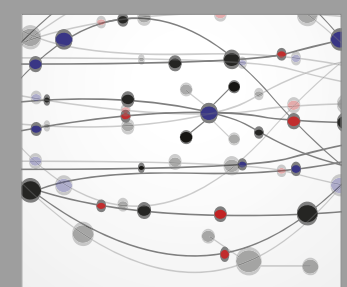

The Scientific World Journal
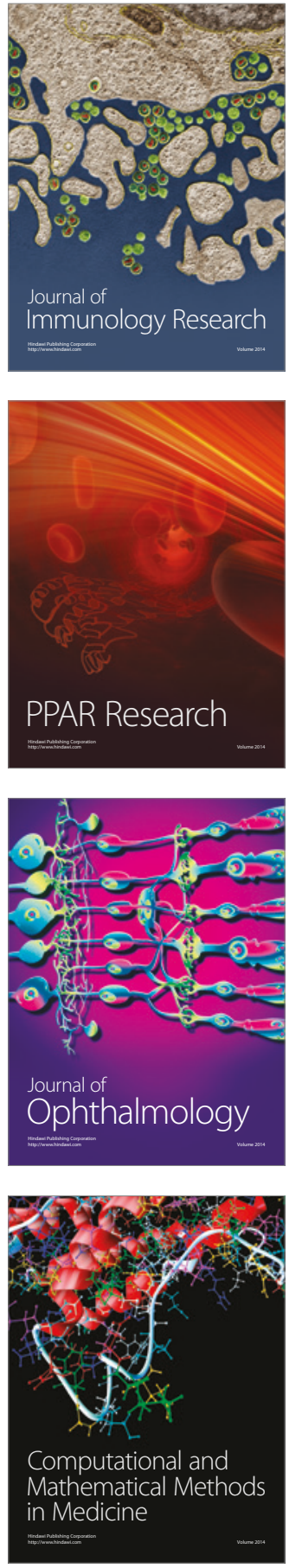

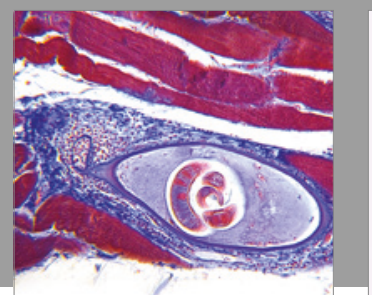

Gastroenterology Research and Practice
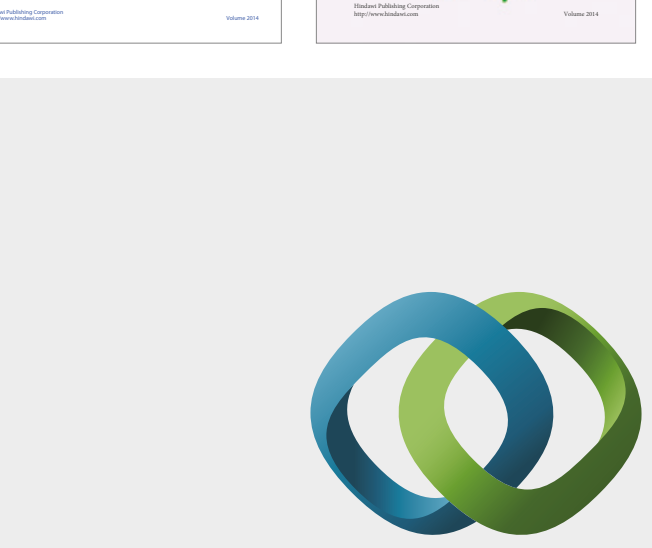

\section{Hindawi}

Submit your manuscripts at

https://www.hindawi.com
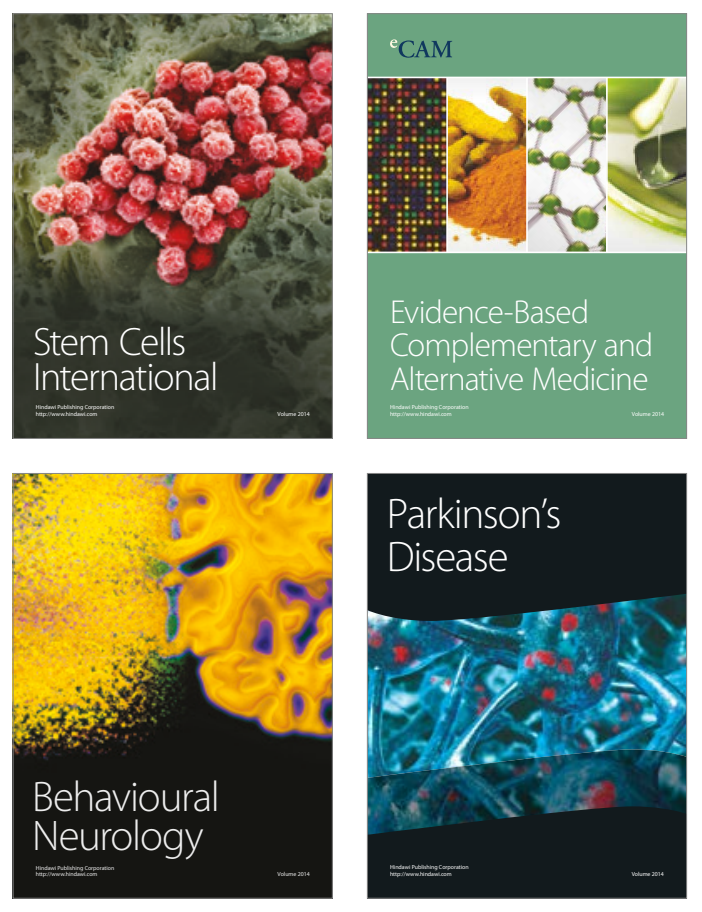
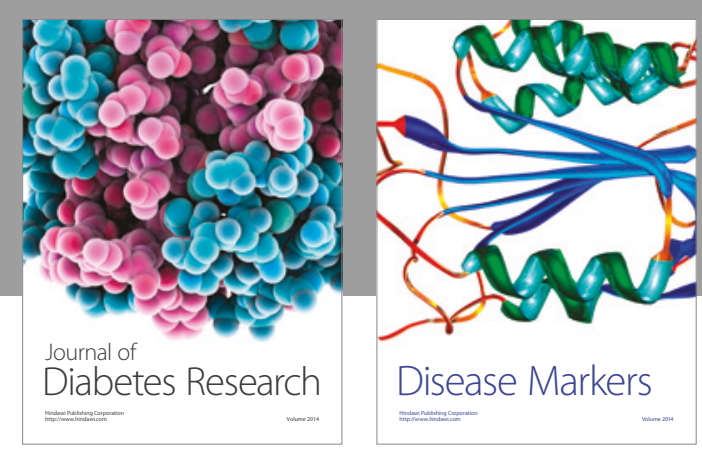

Disease Markers
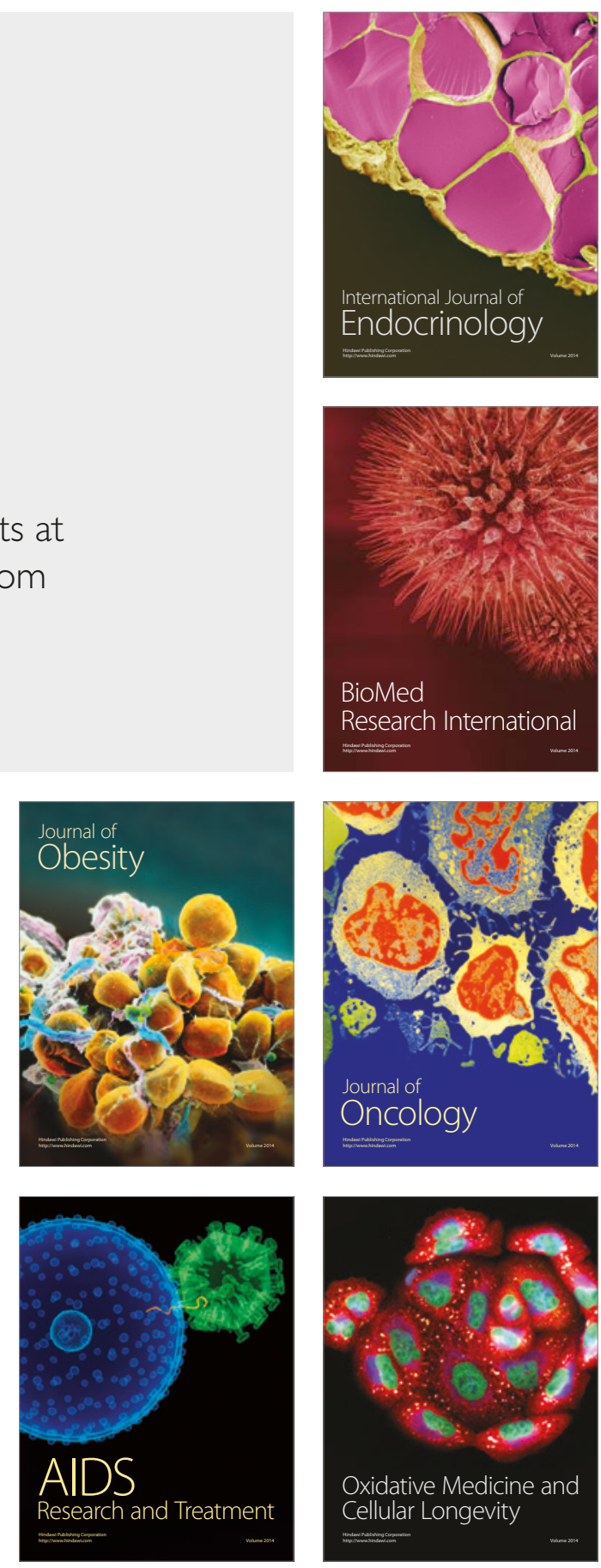